IMPACTOS AMBIENTALES EN EL SECTOR EMPRESARIAL DE LA RECOLECCIÓN CONTRATADA DE RESIDUOS SÓLIDOS URBANOS EN LA CIUDAD DE TEPIC, NAYARIT, MÉXICO.

\title{
IMPACTOS AMBIENTALES EN EL SECTOR EMPRESARIAL DE LA RECOLECCIÓN CONTRATADA DE RESIDUOS SÓLIDOS URBANOS EN LA CIUDAD DE TEPIC, NAYARIT, MÉXICO
}

\section{ENVIRONMENTAL IMPACTS ON THE BUSINESS SECTOR OF THE CONTRACTED COLLECTION OF MUNICIPAL SOLID WASTE IN THE CITY OF TEPIC, NAYARIT, MEXICO}

Ana Griset Tapia Ibarra*, Alicia del Carmen Valencia Ovalle**, Claudia E. Saldaña Durán***

*Estudiante de Doctorado en Gestión de las Organizaciones. Maestra en Desarrollo Económico Local. Universidad Autónoma de Nayarit. Av. Ejército Mexicano esq. Av. Universidad s/n. Ciudad Universitaria. C.P. 82017. México. Email: agti_22@hotmail.com. ORCID: https://orcid.org/0000-0001-8151-4393.

**Dra. en Ciencias para el Desarrollo Sustentable. Cuerpo Académico "Las Organizaciones y su Ambiente", CA-UAN-251. Unidad Académica de Contaduría y Administración. Universidad Autónoma de Nayarit. Ciudad de la Cultura "Amado Nervo". C.P. 63155. México. Email: alvalov@gmail.com. ORCID: https://orcid.org/0000-0001-6902-3559.

***Dra. en Ciudad, Territorio y Sustentabilidad. Cuerpo Académico de "Sustentabilidad "Energética" UANCA-257. Unidad Académica de Ciencias Básicas e Ingenierías. Universidad Autónoma de Nayarit. Ciudad de la Cultura "Amado Nervo". C.P. 63155. México. https://orcid.org/0000-0002-2022-6379.

Dirección para recibir correspondencia: cesduran@uan.edu.mx 
IMPACTOS AMBIENTALES EN EL SECTOR EMPRESARIAL DE LA RECOLECCIÓN CONTRATADA DE RESIDUOS SÓLIDOS URBANOS EN LA CIUDAD DE TEPIC, NAYARIT, MÉXICO.

\section{RESUMEN}

El propósito de este trabajo es implementar el análisis del ciclo de vida en las empresas de recolección de residuos sólidos urbanos, con el propósito de determinar los principales impactos ambientales y, con base en esto, establecer estrategias que mitiguen estos impactos. La implementación del análisis del ciclo de vida en cualquier organización sería una excelente medida de la regulación ambiental en el sector empresarial, proporcionando importantes beneficios sociales y ambientales.

OBJETIVO: Determinar los impactos ambientales derivados de la actividad de una organización dedicada a la recolección contratada de Residuos Sólidos Urbanos (RSU) en la Ciudad de Tepic, Nayarit, México.

MATERIAL Y MÉTODO: Se utilizó la metodología de ACV (Análisis del Ciclo de Vida) siguiendo la norma NMX-SAA-14040 considerando las cuatro fases: a) Definición del objetivo y el alcance; b) Análisis del inventario; c) Evaluación del impacto del ciclo de vida; y la d) Interpretación. Para el proceso de datos se utilizó el programa Simapro Versión 8.5.0.

RESULTADOS: Se logró obtener los impactos ambientales del SERC (Sector Empresarial de la Recolección Contratada) Se realizó un análisis comparativo de las categorías de impacto tomando como referencia dos escenarios: actividad principal de la empresa y el reciclaje de plástico, cartón-papel, vidrio y aluminio; en la evaluación del escenario 2 se obtuvieron resultados favorables en la categoría de impacto "recursos minerales". Se concluye, que el reciclaje debe de ser una medida de regulación ambiental del SERC impactando de forma positiva al medio ambiente y a la reducción significativa de toneladas de $\mathrm{CO}_{2 \text { eq. }}$

CONCLUSIONES: Al llevar a cabo el primer enfoque LCA (Life Cycle Assessment) de la actividad realizada por la organización (colección contratada de RSU), se obtuvo un análisis comparativo de las categorías de impacto tomando como referencia dos escenarios. El primero se realizó tomando como referencia la actividad principal de la organización, mientras que en el segundo escenario se tiene en cuenta el reciclaje de plástico, cartón, papel, vidrio y aluminio; en la evaluación de los escenarios propuestos, se puede apreciar que escenario 2, presenta resultados favorables en la categoría de impacto de "recursos minerales" (escenario $1 \mathrm{~kg} \mathrm{Cu}$ eq = 0.06661551 vs escenario $2 \mathrm{Kg} \mathrm{Cu}$ eq $=-0.03463779$ ). En conclusión, el reciclaje debe ser una medida de la regulación ambiental en el sector empresarial, especialmente en las organizaciones TAPIA-IBARRA A. G., VALENCIA-OVALLE A. DEL C., SALDAÑA-DURÁN C. E. 
IMPACTOS AMBIENTALES EN EL SECTOR EMPRESARIAL DE LA RECOLECCIÓN CONTRATADA DE RESIDUOS SÓLIDOS URBANOS EN LA CIUDAD DE TEPIC, NAYARIT, MÉXICO.

de recolección contratadas, impactando positivamente el medio ambiente al reducir significativamente las toneladas de $\mathrm{CO} 2$ eq. de gases de efecto invernadero.

PALABRAS CLAVE: Análisis de ciclo de vida. Reciclaje. Impactos ambientales. Residuos sólidos.

\section{ABSTRACT}

The purpose of this work is to implement the life cycle analysis in urban solid waste collection companies, with the purpose of determining the main environmental impacts and on this basis, establish strategies that mitigate these impacts. Implementing the life cycle analysis in any organization would be an excellent measure of environmental regulation in the business sector, providing important social and environmental benefits.

OBJECTIVE: To determine the environmental impacts derived from the activity of an organization dedicated to the contracted collection of Municipal Solid Waste (MSW) in the city of Tepic, Nayarit, Mexico.

MATERIALS AND METHODS: The LCA (Life Cycle Assessment) methodology was used following the NMX-SAA-14040 standard, which considered the four phases that consist of: a) Definition of the objective and scope; b) Inventory analysis; c) Life cycle impact assessment; and d) Interpretation. For data processing the Simapro Version 8.5.0 program was used.

RESULTS: The environmental impacts of the SERC (Sector Empresarial de la Recolección Contratada) were obtained. A comparative analysis of the impact categories was carried out taking two scenarios as a reference: the company's main activity and the recycling of plastic, cardboardpaper, glass and aluminum; In the evaluation of the scenario 2, favorable results were obtained in the impact category "mineral resources". It is concluded that recycling should be an environmental regulation measure of the SERC, positively impacting the environment and the significant reduction of tons of CO2eq. 
IMPACTOS AMBIENTALES EN EL SECTOR EMPRESARIAL DE LA RECOLECCIÓN CONTRATADA DE RESIDUOS SÓLIDOS URBANOS EN LA CIUDAD DE TEPIC, NAYARIT, MÉXICO.

CONCLUSIONS: When carrying out the first LCA approach of the activity accomplished by the organization (contracted collection of MSW), a comparative analysis of the impact categories was obtained taking two scenarios as a reference. The first one was carried out only taking as reference the main activity of the organization, while in the second scenario, the recycling of plastic, cardboard-paper, glass and aluminum is taken into account; It can be seen that in the evaluation of the proposed scenarios, scenario 2, presents favorable results in the "mineral resources" impact category (scenario $1 \mathrm{~kg} \mathrm{Cu} \mathrm{eq}=0.06661551$ vs scenario $2 \mathrm{Kg} \mathrm{Cu}$ eq = 0.03463779). In conclusion, recycling must be a measure of environmental regulation in the business sector, especially in contracted collection organizations, positively impacting the environment by significantly reducing CO2eq tons. of greenhouse gases.

KEYWORDS: Life cycle analysis. Recycling. Environmental impacts. Solid waste.

\section{INTRODUCCIÓN}

La intensificación de la industrialización que se presentó en México durante la segunda mitad del siglo pasado, produjo una mayor demanda de materias primas para satisfacer el creciente consumo de bienes y servicios de una población en aumento y con patrones de consumo cambiantes y cada vez más demandantes. A la par, creció la generación de residuos de distintos tipos y los problemas asociados para su adecuada disposición, así como las afectaciones a la salud humana y a los ecosistemas (SEMARNAT, 2012). Debido a la rápida urbanización, la generación de residuos sólidos municipales aumenta en gran medida, siendo inevitable el aumento de estos a lo largo de los años; se calcula que dicha generación se incrementa en más del 90\% por cada 10 años (Murad \& Siwar, 2007 citado en Moh \& Manaf, 2017).

El Banco Mundial en su estudio Una Revisión Global de Gestión de Residuos Sólidos (2012), "estimó que de los 3,000 millones de residentes urbanos que existían a esa fecha generaron 1.20 kilos de basura per cápita al día, es decir 1.3 millones de toneladas anuales, con una proyección para el 2025 probable de 4.3 mil millones de residentes urbanos que generarán aproximadamente, 1.42 kilos per cápita al día, esto es, 2,200 millones de toneladas anuales". Entre las causas de este incremento, se mencionan el alto crecimiento poblacional, los hábitos TAPIA-IBARRA A. G., VALENCIA-OVALLE A. DEL C., SALDAÑA-DURÁN C. E. 
IMPACTOS AMBIENTALES EN EL SECTOR EMPRESARIAL DE LA RECOLECCIÓN CONTRATADA DE RESIDUOS SÓLIDOS URBANOS EN LA CIUDAD DE TEPIC, NAYARIT, MÉXICO.

de consumo en países industrializados, así como los cambios en las costumbres de consumidores que habitan los países en vía de desarrollo.

En este mismo sentido, Hoornweg \& Bhada (2012), han señalado que países miembros de la Empresa para la Cooperación y el Desarrollo Económico (OCDE) son los mayores generadores de residuos, produciendo alrededor de 1.75 millones de toneladas por día. En contraste, se tiene el caso de Japón, el cual genera aproximadamente un tercio menos de residuos por habitante que Estados Unidos, a pesar de tener un producto interno bruto (PIB) per cápita similar; mientras que en China la generación de residuos sólidos en el año 2005 fue de 520,550 toneladas por día y se espera que se incremente a 1.4 millones de toneladas diarias en 2025 (Parada y Gutiérrez, 2015).

Para el caso de América Latina y el Caribe, a través de la evaluación de la gestión de residuos sólidos (Tello et al., 2010), estimó que la generación per cápita de residuos sólidos domiciliarios (RSD) alcanza $0.63 \mathrm{~kg} / \mathrm{hab} /$ día, mientras que la de residuos sólidos urbanos (RSU) asciende a $0.93 \mathrm{~kg} / \mathrm{hab} / \mathrm{día}$. Los indicadores per cápita obtenidos para la región implican una generación urbana diaria aproximada de 295,000 ton de RSD y 436,000 de RSU. La Evaluación de la Gestión de Residuos Sólidos Urbanos en América Latina y el Caribe (EVAL), estimó que los residuos recolectados en América Latina y el Caribe transferidos disminuyó entre 2002 y 2010 de 37.9\% a 28.2\%; aunque la incorporación de países como Brasil y Colombia mejoró la representatividad de la estadística en gran medida. Entre las principales alternativas de tratamiento de los residuos antes de su disposición final se pueden mencionar el compostaje, reciclaje y tratamiento térmico, incluyendo en algunos casos el aprovechamiento energético de los desechos, si bien estas actividades son todavía incipientes en América Latina y el Caribe, son ampliamente usadas en los países desarrollados (Tello et al., 2010).

En este mismo contexto, para México se estima que cada habitante genera alrededor de $900 \mathrm{gr}$ de residuos al día, generación que va desde $400 \mathrm{gr}$ en zonas rurales, hasta cerca de $1.5 \mathrm{~kg}$ en zonas metropolitanas. De esta forma, se calcula que en el país se producen cerca de 100,800 toneladas de "basura" al día, lo que significa cerca de 37 millones de toneladas de residuos al año. En la composición de estos residuos, se estima que el 53\% son residuos orgánicos biodegradables, $14 \%$ papel y cartón, $6 \%$ vidrio, $4 \%$ plásticos, $3 \%$ metales y $1 \%$ textiles, mientras que el restante (19\%) corresponde a otro tipo de materiales. Sin embargo, a pesar de las posibles ventajas económicas y ambientales que representaría la recuperación de estos materiales, se TAPIA-IBARRA A. G., VALENCIA-OVALLE A. DEL C., SALDAÑA-DURÁN C. E. 
IMPACTOS AMBIENTALES EN EL SECTOR EMPRESARIAL DE LA RECOLECCIÓN CONTRATADA DE RESIDUOS SÓLIDOS URBANOS EN LA CIUDAD DE TEPIC, NAYARIT, MÉXICO.

estima que solo se recupera para su comercialización entre el 10 y el 12\% del total generado y desafortunadamente, en la mayoría de los casos, los residuos se convierten en "basura" debido a que estos se disponen mezclados en el mismo contenedor o en un mismo sitio con la correspondiente dificultad para su debida recuperación (SEMARNAT, 2010).

Durante el año 2012, en México se generaron 42.1 millones de toneladas de RSU anuales, 15\% más que en el año 2000. En los últimos años la generación de estos residuos ha incrementado en más del 90\% pasando de 21.9 millones de toneladas en 1992 a 41.1 millones de toneladas en el 2012 (SEMARNAT, 2012). Pudiéndose observar que el mayor crecimiento en la generación de residuos es experimentado en las ciudades medias y en las zonas metropolitanas, localidades que han presentado esta tendencia desde el 2001. Esta situación afirma lo señalado a niveles internacionales, sobre la relación que existe entre las grandes ciudades y una mayor producción de residuos sólidos en comparación con las ciudades pequeñas. Por otra parte, en cuanto a la generación per cápita, los datos permiten observar que dicho indicador en 2012 fue de 1 kg/hab/día en los municipios mayores a 100 mil habitantes, es decir, en estos espacios urbanos la generación por persona fue superior a la media nacional (Jiménez, 2015).

\section{Estrategias para la gestión integral de los residuos sólidos urbanos desde tres contextos (mundial, latinoamericano y nacional)}

Ante esta situación, el Programa de las Naciones Unidas para el Medio Ambiente (PNUMA), publicó un estudio denominado Perspectivas del Medio Ambiente Mundial (GEO-5), argumentando que la directriz sobre el control de los desechos está enfocada al reuso, el reciclaje y la recuperación (PNUMA, 2012). En relación con lo anterior se presentan las estrategias que han seguido a nivel mundial, Latinoamérica y México (ver tabla 1). 
IMPACTOS AMBIENTALES EN EL SECTOR EMPRESARIAL DE LA RECOLECCIÓN CONTRATADA DE RESIDUOS SÓLIDOS URBANOS EN LA CIUDAD DE TEPIC, NAYARIT, MÉXICO.

Tabla 1

Estrategias en el tratamiento de residuos sólidos urbanos

\begin{tabular}{|c|c|c|c|}
\hline Nivel & País & $\begin{array}{c}\text { Estrategias en el } \\
\text { tratamiento de residuos }\end{array}$ & Empresas Recicladoras \\
\hline Mundial & $\begin{array}{l}\text { Unión } \\
\text { Europea, } \\
\text { India, } \\
\text { Brasil }\end{array}$ & $\begin{array}{l}\text { Tratamiento adecuado de } \\
\text { los residuos electrónicos, } \\
\text { así como un destino } \\
\text { especial por el contenido de } \\
\text { plomo y mercurio (Parada y } \\
\text { Gutiérrez, 2015; Echegaray } \\
\text { \& Hansstein, 2017). }\end{array}$ & $\begin{array}{l}\text { No hay datos precisos. } \\
12 \text { asociaciones: ARpAL, } \\
\text { ASEGRE, ANFAC, } \\
\text { AEDRA ,BIR, EFR y Eurometrec, } \\
\text { UNESID, REpACAR, ASERMA, } \\
\text { ANARpLA, FEAF. }\end{array}$ \\
\hline Latinoamérica & Brasil & $\begin{array}{l}\text { El reciclaje y el compostaje } \\
\text { son programas clave para } \\
\text { reducir el impacto ambiental } \\
\text { (Mattos, Gomes \& Ribeiro, } \\
\text { 2017). }\end{array}$ & $\begin{array}{l}1,000 \text { empresas del SERC; } 90 \% \\
\text { de los materiales reciclados son } \\
\text { recogidos por recolectores } \\
\text { informales, solo el } 2.2 \% \text { es } \\
\text { recuperado formalmente y } \\
\text { reciclado (Avina, 2012). }\end{array}$ \\
\hline México & Nacional & $\begin{array}{lr}\text { Aplicar la filosofía de las } \\
\text { 3Rs. Promover la } \\
\text { minimización, } \\
\text { aprovechamiento y la } \\
\text { valorización de los residuos. } \\
\text { Programa Nacional para la } \\
\text { Prevención y Gestión } \\
\text { Integral de los Residuos } \\
\text { (SEMARNAT, 2008). }\end{array}$ & $\begin{array}{l}23 \text { plantas de selección INECC- } \\
\text { SEMARNAT (2012). }\end{array}$ \\
\hline
\end{tabular}

Nota: Según el organismo de la EVAL determina que varios países han hecho progresos significativos en la gestión de los residuos sólidos urbanos en los últimos ocho años, mostrando que algunas de las actividades de la gestión de residuos en ALC son incipientes, por ejemplo, las relacionadas con la reducción, recuperación y reciclaje de residuos; además de los beneficios en la recuperación de biogás. Pero, por otra parte, se muestra un atraso en áreas relacionadas con la recolección selectiva, compostaje, reciclado de materiales y tratamiento térmico de residuos con recuperación de energía (Tello et al., 2010).

Fuente: Elaboración propia con base a los documentos citados en la tabla.

El tratamiento de los residuos es más factible a partir de su separación, en América Latina y el Caribe (ALC), el reciclaje formal en plantas de separación es casi inexistente en la actualidad. El reciclaje informal, por su parte, está muy extendido, pero se desconocen con precisión las cantidades recicladas. El compostaje, emprendido en numerosas oportunidades en ALC, podría recibir un fuerte impulso con el uso de fondos de carbono. La incineración casi no ha sido utilizada en ALC, pero tecnologías más eficientes de tratamiento térmico con aprovechamiento energético 
IMPACTOS AMBIENTALES EN EL SECTOR EMPRESARIAL DE LA RECOLECCIÓN CONTRATADA DE RESIDUOS SÓLIDOS URBANOS EN LA CIUDAD DE TEPIC, NAYARIT, MÉXICO.

de los residuos pueden representar una opción de tratamiento válida en grandes ciudades que debe estudiarse en cada caso (Tello et al., 2010).

\section{La importancia del reciclaje desde la perspectiva económica y ambiental}

La industria del reciclaje es fuente importante de ingresos para una extensa cadena productiva, desde aquellas personas que realizan el acopio hasta las empresas que venden la materia prima derivada de los productos reciclables; sin embargo, la carencia de un esquema fiscal que dé certeza a las compras de residuos propicia que millones de pesos salgan del país principalmente con dirección al continente asiático, para regresar en una gran variedad de productos terminados que son consumidos en México. A falta de este esquema, la inversión privada se aleja, sólo en 2011 dejaron de ingresar al país inversiones por unos cinco millones de dólares, que servirían para la apertura de plantas y dar salida a las mil 200 toneladas de residuos plásticos que se generan a diario y que hasta hoy se van a los rellenos sanitarios (ANIPAC, 2012).

El mercado está comenzando a percibir el uso de materiales reciclados como una ventaja, gracias a tecnologías para hacer nuevos productos de alto valor; el reciclaje de Polietileno de Tereftalato (PET) se está proyectando como parte fundamental del boom ambiental que se vive actualmente, convirtiéndose en una de las mayores oportunidades de negocios para toda la industria del plástico, pero a pesar de que se exalta la reciclabilidad del PET como una ventaja ambiental y económica en la industria, una gran cantidad de este material sigue sin ser recuperado, el cual se destina a los rellenos sanitarios o vertederos superando a la cantidad que se recicla (Moh \& Manaf, 2017).

Por ejemplo, Japón, el país más avanzado en este campo, registró una tasa de reciclaje de botellas de PET de 77.9 \% en 2009 (volumen total de recolección/volumen de botellas vendidas), según datos del Consejo de Reciclaje de Botellas de PET de ese país. Por su parte, Europa tiene cifras consolidadas de 48.3\%, de acuerdo con la Asociación Europea de Reciclaje de Contenedores de PET (Petcore); Estados Unidos de 28\% según la Asociación de Resinas de PET (Petra); mientras que Brasil es el líder con una tasa de 55.6\%, seguido por Argentina con 34\%, según reportes de la Asociación Brasileña de la Industria del PET (ABIPET) (Ortega, 2011).

Mercados como el europeo y el estadounidense, con tasas de reciclaje superiores al 40\%, tienen un valor de más de 70 mil millones de dólares, con crecimientos sostenidos del 11\% en promedio anual. Promotora Ambiental (PASA), que cotiza en la Bolsa Mexicana de Valores (BMV), y TAPIA-IBARRA A. G., VALENCIA-OVALLE A. DEL C., SALDAÑA-DURÁN C. E. 
IMPACTOS AMBIENTALES EN EL SECTOR EMPRESARIAL DE LA RECOLECCIÓN CONTRATADA DE RESIDUOS SÓLIDOS URBANOS EN LA CIUDAD DE TEPIC, NAYARIT, MÉXICO.

Proactiva, en la que Ingenieros Civiles Asociados (ICA) tenían participación accionaria; dedican sus esfuerzos a la recolección, tratamiento y reciclaje de residuos, los cuales posteriormente comercializan e incorporan nuevamente al proceso productivo. En 2015, PASA generó ingresos superiores a los 3 mil millones de pesos, y al cierre del segundo trimestre de 2016 su negocio de manejo de residuos creció 10.2\%, respecto al 2015 al facturar 851 millones (El Financiero, 2016).

En este mismo orden de ideas, la mayoría de las ciudades latinoamericanas no recolecta la totalidad de los desechos sólidos generados, sólo una fracción de los desechos recibe una disposición final adecuada, provocando contaminación ambiental y riesgos para la salud humana. El reciclaje representa una opción deseable en lugar de la disposición masiva de desechos en basureros o rellenos sanitarios, sin embargo, pocos programas oficiales de reciclaje existen en dichas ciudades latinoamericanas. El reciclaje ha ganado aceptación y popularidad, como una forma de disminuir la cantidad de residuos que necesitan disposición final en rellenos sanitarios, así como de reducir el impacto ambiental negativo de las actividades productivas y de consumo (Medina, 1999; Tisserant et al., 2017; Mattos, Gomes \& Ribeiro, 2017).

En el caso de México, sus entidades federativas recolectan menos del $75 \%$ de los residuos generados (Medina, 2005), según datos del Instituto Nacional de Estadística y Geografía (INEGI, 2012), apenas se reutilizaba el $11 \%$ de las 86 mil toneladas de residuos que se generan al día en el país, el cual tiene un valor potencial de 3 mil millones de dólares, con un crecimiento promedio anual del 10\%, de acuerdo con estimaciones de la Asociación Nacional de Industrias del Plástico (ANIPAC). Así mismo, el no reciclar genera un "costo de degradación ambiental superior a 61 millones de pesos y un costo en salud pública mayor a 32 millones de pesos anualmente" (Milenio Digital, 2017).

En relación con la recuperación y comercialización de material reciclable post consumo, la clasificación y minimización de los RSU se hace cada vez más necesaria en México; ya que el incremento poblacional y la urbanización acelerada del país, han ocasionado un flujo de basura incontrolado en los municipios, provocando costos sociales y económicos crecientes asociados a su recolección, manejo y disposición final (Castillo, 1983; Buenrostro et al., 2001; Castillo, 2003). Por otra parte, la demanda creciente de los recursos naturales renovables está obligando a que éstos sean utilizados de manera racional y sostenida para evitar su agotamiento. En los RSU existen numerosos subproductos que pueden ser nuevamente utilizados como materia prima, al retirar los materiales reutilizables o reciclables del flujo de la basura, se disminuye el TAPIA-IBARRA A. G., VALENCIA-OVALLE A. DEL C., SALDAÑA-DURÁN C. E. 
IMPACTOS AMBIENTALES EN EL SECTOR EMPRESARIAL DE LA RECOLECCIÓN CONTRATADA DE RESIDUOS SÓLIDOS URBANOS EN LA CIUDAD DE TEPIC, NAYARIT, MÉXICO.

volumen y la cantidad de los desperdicios que son enviados a disposición final, lo cual resulta beneficioso para el medio ambiente. Por ello, se quiere que la minimización (reducción), el reúso y el reciclaje sean las actividades estratégicas para la eliminación de la basura doméstica (Tonglet et al., 2004 citado en Maldonado, 2006).

Es fundamental señalar que el proceso de reciclaje ayuda al ahorro de recursos naturales, disminución de la contaminación, alarga la vida de los materiales, ahorra energía y reduce hasta en un $80 \%$ el espacio que ocupan los residuos dentro de los rellenos sanitarios (Michell, 2007; Mattos, Gomes \& Ribeiro, 2017). Cabe destacar que el 90\% de los residuos sólidos inorgánicos se pueden reciclar, es decir, los productos de desecho pueden ser nuevamente utilizados, ya sea mediante un proceso de reciclaje mecánico o químico (Frers, 2005 citado en Torres, Salas y García, 2010).

Evidentemente la creciente generación de residuos sólidos urbanos es algo inevitable por la relación directa con el patrón de consumo y el crecimiento demográfico, generando la problemática de no poder dar un manejo adecuado a los desechos, por lo cual se considera de vital importancia fomentar en las empresas recolectoras de Residuos Sólidos (RS) la implementación de enfoques de gestión ambiental, que junto a las tecnologías alternativas, participación social y educación ambiental, serían claves para el manejo adecuado de dichos desechos, tomando en consideración las etapas del ciclo de vida del producto-residuo, desde la producción (elección de materiales y procesos) hasta su reinserción en un nuevo ciclo de vida a través de la reutilización y reciclaje (Garcí y Salgado, 2007).

\section{Operaciones de la actividad de recuperación de residuos susceptibles de producir impacto ambiental}

La actividad de recuperación de los residuos conlleva las siguientes operaciones que pueden producir impactos ambientales: 1. Manejo de los residuos en la fuente de generación; 2. Traslado de los residuos recuperables a los centros de transferencia; 3. Descarga y carga de los residuos recuperables en los centros de acopio; 4. Clasificación de los residuos recuperables en los centros de acopio; 5. Almacenamiento de los residuos recuperables en los centros de acopio; 6. Carga de los vehículos de recolección en los centros de acopio; 7. Transporte de los residuos recuperables de los centros de acopio a las industrias recicladoras (Salas y Quesada, 2006).

El análisis de ciclo de vida como una técnica de evaluación en los impactos ambientales TAPIA-IBARRA A. G., VALENCIA-OVALLE A. DEL C., SALDAÑA-DURÁN C. E. 
IMPACTOS AMBIENTALES EN EL SECTOR EMPRESARIAL DE LA RECOLECCIÓN CONTRATADA DE RESIDUOS SÓLIDOS URBANOS EN LA CIUDAD DE TEPIC, NAYARIT, MÉXICO.

El ACV es un marco metodológico para la estimación y evaluación de los impactos potenciales sobre el medio ambiente, atribuibles al ciclo de vida de un producto, es decir, desde la adquisición de materias primas a través de las fases de producción y el uso, hasta la gestión de residuos" (Rebitzer et al, 2004 citado en Vélez, 2014; Olivera, Cristobal y Saizar, 2016; ISO 14044, 2006). Permite evaluar las cargas ambientales asociadas a un producto, proceso 0 actividad, identificando y cuantificando tanto el uso de materia y energía como las emisiones al entorno, para poder así determinar su impacto, llevar a la práctica estrategias de mejora ambiental y realizar declaraciones ambientales (Olivera, Cristobal y Saizar, 2016).

Por lo anterior, el objetivo principal de la investigación es determinar los impactos ambientales derivados del SERC de RSU en la Ciudad de Tepic, Nayarit, México; través de la implementación de la metodología del Análisis de Ciclo de Vida. Se tomó en consideración la norma NMX-SAA14040 (IMNC, 2008), para realizar las cuatro fases del análisis de ciclo de vida (ver figura 1).

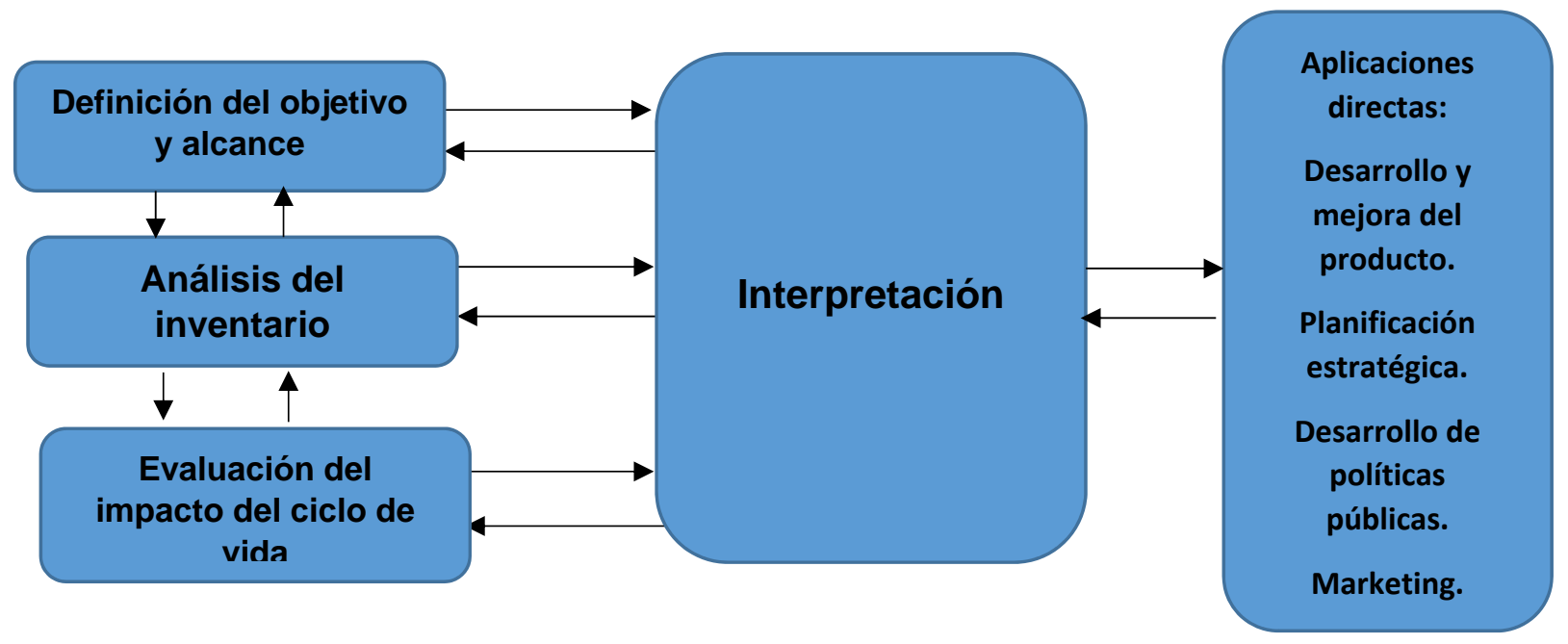

Figura 1. Etapas del Análisis del ciclo de vida según la norma NMX-SAA-14040. Fuente: Elaboración propia.

De acuerdo con Olivera, Cristobal y Saizar (2016), en la primera fase se establecen los objetivos y cometidos del estudio, su alcance se determina con los límites fijados para el sistema, la unidad funcional y los flujos dentro del ciclo de vida. La segunda fase corresponde al análisis de inventario, en la que se recopilan los datos correspondientes a las entradas y salidas para todos los procesos del sistema en estudio, los principales datos recogidos corresponden con entradas de energía, entradas de materia prima, entradas auxiliares, productos, co-productos y residuos, 
IMPACTOS AMBIENTALES EN EL SECTOR EMPRESARIAL DE LA RECOLECCIÓN CONTRATADA DE RESIDUOS SÓLIDOS URBANOS EN LA CIUDAD DE TEPIC, NAYARIT, MÉXICO.

emisiones y otros aspectos ambientales, entre otros. Respecto a la Evaluación de Impacto del Ciclo de Vida (EICV), el objetivo principal de esta fase es proporcionar información para abordar el cálculo que permita evaluar los resultados del inventario del ciclo de vida de un sistema y detectar los impactos ambientales potenciales, mientras que la interpretación de resultados es la fase en la que se discuten los resultados de análisis del inventario y/o evaluación de impacto. Los resultados de esta interpretación son la base para obtener las conclusiones, recomendaciones y toma de decisiones de acuerdo con el objetivo y alcance definidos.

\section{MATERIAL Y MÉTODO}

La ciudad de Tepic, Nayarit, lugar donde se llevó a cabo la investigación, cuenta con tres organizaciones con la característica en común de ofrecer el servicio de recolección contratada de residuos sólidos urbanos. Para elegir la empresa en la que se realizó la intervención, se tomaron los siguientes criterios de inclusión: 1) Proporcionar el servicio de recolección contratada de residuos sólidos urbanos; 2) Número de clientes atendidos; 3) Giros a quienes otorgan el servicio y 4) Contar con centro de transferencia. Por lo anterior, REDESO fue la empresa seleccionada ya que cubre con todos los criterios de inclusión mencionados, además de ser una empresa que a partir del año 2012, comenzó con la separación de residuos con valor económico, siendo los principales materiales separados, diversos tipos de plásticos (PET, HDPE, LDPE, PP), cartón/papel, aluminio y vidrio (color y transparente).

Con el fin de evaluar la carga ambiental del proceso de la recolección contratada de los residuos sólidos urbanos, se llevó a cabo un ACV conforme a la norma mexicana NMX-SAA-14040. Esta metodología presenta un enfoque holístico para estimar los impactos ambientales relacionados con todo el ciclo de vida de un producto o servicio. Los límites del estudio comprendieron la cadena que se extiende desde la recolección de los residuos hasta su confinamiento en el relleno sanitario el Iztete de la Ciudad de Tepic, Nayarit, México.

El software elegido para la realización del estudio es el programa SimaPro 8.5, el cual permite calcular los impactos ambientales, sociales y económicos asociados a un producto a lo largo de todo su ciclo de vida. Este software contiene bases de datos que incluyen varias librerías o proyectos que, a su vez, agrupan diferentes procesos con todas sus entradas y salidas. Los procesos se separan en siete categorías: materiales, energía, transporte, procesado, uso, escenario de residuos y tratamiento de residuos. Además, la base de datos contiene entradas de 
IMPACTOS AMBIENTALES EN EL SECTOR EMPRESARIAL DE LA RECOLECCIÓN CONTRATADA DE RESIDUOS SÓLIDOS URBANOS EN LA CIUDAD DE TEPIC, NAYARIT, MÉXICO.

carácter general como son los nombres de sustancias, las unidades de medida, referencias de la literatura, entre otros.

La metodología de evaluación de impacto seleccionada para el presente estudio, fue la $\operatorname{ReCiPe}^{1}$ la cual se desarrolló para combinar las ventajas de los métodos CML2001² y Eco-Indicator99. La ventaja del método CML es su solidez científica, mientras que la ventaja del Eco-indicator 99 es su facilidad de interpretación, esta metodología integra el enfoque orientado al problema ambiental y el orientado al daño, comprende, por tanto, dos grupos de categorías de impacto: uno de puntos intermedios, que incluye 18 categorías (Cambio climático, Disminución de la capa de ozono, Toxicidad humana, Formación de oxidantes fotoquímicos, Formación de materia particulada, Radiación ionizante, Acidificación terrestre, Eutrofización de agua dulce, Eutrofización marina, Ecotoxicidad terrestre, Ecotoxicidad de agua dulce, Ecotoxicidad marina, Ocupación de terreno agrícola, Ocupación de terreno urbano, Transformación de terreno natural, Disminución de cantidad de agua dulce, Disminución de recursos minerales y Disminución de combustibles fósiles) y otro de puntos finales que incluye 3 categorías (salud humana, ecosistemas y aumento del costo de recursos).

Los datos que no se encuentran contenidos dentro de las librerías del software seleccionado, para realizar el análisis de los impactos ambientales, se obtuvieron mediante la aplicación de una encuesta diseñada específicamente para recabar la información, la encuesta se aplicó a personal clave de la empresa en donde se tomaron en consideración variables financieras, ambientales y operativas. Se revisó la validez y confiabilidad del instrumento mediante la revisión por parte de expertos (validez de contenido), y el análisis de confiabilidad mediante el coeficiente Alfa de Cronbach, obteniendo un resultado de 0.896 .

\section{RESULTADOS Y DISCUSIÓN}

Siguiendo las cuatro etapas del Análisis de Ciclo de Vida, se encuentra lo siguiente:

\footnotetext{
${ }^{1}$ El sofware SIMAPRO Versión 8.5.0. utiliza un modelo para medir los impactos del ciclo de vida comúnmente utilizado ReCiPe. El ReCiPe proporciona un método de evaluación de impacto de última generación para convertir los inventarios del ciclo de vida en una cantidad de puntajes de impacto armonizados a nivel de punto medio y punto final.

${ }^{2}$ La metodología de CML, elaborada por el Instituto de Ciencias Medioambientales de la Universidad de Leiden en los Países Bajos, es la metodología más utilizada y que suele considerarse más completa. Para derivar los factores de impacto utiliza fundamentalmente datos europeos. TAPIA-IBARRA A. G., VALENCIA-OVALLE A. DEL C., SALDAÑA-DURÁN C. E. 
IMPACTOS AMBIENTALES EN EL SECTOR EMPRESARIAL DE LA RECOLECCIÓN CONTRATADA DE RESIDUOS SÓLIDOS URBANOS EN LA CIUDAD DE TEPIC, NAYARIT, MÉXICO.

\section{Etapa 1. Límites del Sistema y Unidad Funcional}

Límite por tipo de residuos: En este estudio se consideran los denominados Residuos Sólidos Municipales. Residuos procedentes de los domicilios, comercios y oficinas de acuerdo a la clasificación de Wamsler (2000).

Límite espacial: La investigación se limita a la Ciudad de Tepic, Nayarit; con una superficie de 1,657.25 Km² (INAFED, 2018), con una población de 332,863 habitantes (INEGI, 2015); que presenta una tasa de generación de residuos municipales de 0.669 kg/hab/día (INECC, SEMARNAT, 2012). Lo que correspondería a 222.69 toneladas diarias aproximadamente. De las cuales la empresa recolecta 9,744 toneladas al año.

Unidad Funcional: La unidad funcional considerada en este caso, es la gestión de 812 toneladas de residuos sólidos municipales basados en la clasificación de Wamsler (2000). Por lo que la unidad funcional para este caso sería, gestionar los residuos sólidos recolectados por la empresa REDESO durante un mes. Teniendo como flujo de referencia (F.R.) 812 toneladas de residuos.

\section{Etapa 2. Inventario de ciclo de vida escenario 1}

El inventario es la plataforma donde se recopilan las entradas y salidas para el análisis. Es la fase correspondiente a la recopilación y la cuantificación de las entradas y salidas de un sistema durante su ciclo de vida (Olivera, Cristobal y Saizar, 2016).

Tabla 2

Inventario escenario 1

\begin{tabular}{|c|c|c|c|c|c|c|c|}
\hline \multirow{2}{*}{ Etapa } & \multirow{2}{*}{ Proceso } & \multicolumn{3}{|c|}{ Entrada } & \multicolumn{3}{|c|}{ Salida } \\
\hline & & Tipo & Cantidad & Unidad & Tipo & Cantidad & Unidad \\
\hline \multirow{6}{*}{ Generación } & \multirow{2}{*}{ Recolección } & RSM & 812 & Ton. & Lixiviado & 0.5 & $\begin{array}{c}\mathrm{m} 3 / \mathrm{lt} / \mathrm{ton} \\
\text { tratada }\end{array}$ \\
\hline & & & & & DQO & $3,203,750$ & $\mathrm{Mg}$ \\
\hline & \multirow{3}{*}{$\begin{array}{l}\text { Puntos de } \\
\text { recolección- centro } \\
\text { transferencia- } \\
\text { disposición final }\end{array}$} & Diesel & $5,053.08$ & Kg. & COT & $1,581,650$ & $\mathrm{Mg}$ \\
\hline & & $\begin{array}{c}\text { Gasolina } \\
\text { magna }\end{array}$ & 627.30 & $\mathrm{Kg}$. & $\begin{array}{c}\text { Sólidos } \\
\text { suspendidos }\end{array}$ & $2,425,000$ & $\mathrm{Mg}$ \\
\hline & & $\begin{array}{l}\text { (Toneladas } \\
\text { residuos)(km } \\
\text { recorridos) }\end{array}$ & $24,231,622.80$ & Ton/Km & $\mathrm{CO}_{2 \text {-eq }}$ & 970.11* & Ton. \\
\hline & $\begin{array}{l}\text { Servicio de } \\
\text { recolección }\end{array}$ & $\begin{array}{c}\text { Energía } \\
\text { alterna }\end{array}$ & 323.69 & Kwh & & & \\
\hline
\end{tabular}

Fuente: Elaboración propia con base a datos obtenidos de la empresa, así como de fuentes secundarias. TAPIA-IBARRA A. G., VALENCIA-OVALLE A. DEL C., SALDAÑA-DURÁN C. E. 
IMPACTOS AMBIENTALES EN EL SECTOR EMPRESARIAL DE LA RECOLECCIÓN CONTRATADA DE RESIDUOS SÓLIDOS URBANOS EN LA CIUDAD DE TEPIC, NAYARIT, MÉXICO.

Los datos calculados están con base a 8 rutas realizadas por la organización, el cálculo de las emisiones de GEI se determinaron con base a 378,930 ton/a $\mathrm{CO}_{2}$-eq (378.93 $\mathrm{Gg}$ ) para una generación de 317,173 ton/a de RSM. La categoría de residuos representa el 5.9\% de las emisiones de GEI en términos de $\mathrm{CO}_{2}$-eq. (Salmerón et al., 2017).

Tabla 3

Inventario escenario 2

\begin{tabular}{|c|c|c|c|c|c|c|c|}
\hline \multirow[b]{2}{*}{ Etapa } & \multirow[b]{2}{*}{ Proceso } & \multicolumn{3}{|c|}{ Entrada } & \multicolumn{3}{|c|}{ Salida } \\
\hline & & Tipo & Cantidad & Unidad & Tipo & Cantidad & Unidad \\
\hline \multirow{7}{*}{ Generación } & \multirow[b]{2}{*}{ Recolección } & RSM & 812 & Ton. & Lixiviado & 0.5 & $\mathrm{~m}^{3} / \mathrm{l} /$ ton \\
\hline & & & & & DQO & $3,203,750$ & $\begin{array}{c}\text { tratada } \\
\text { Mg }\end{array}$ \\
\hline & \multirow{4}{*}{$\begin{array}{l}\text { Puntos de } \\
\text { recolección- centro } \\
\text { transferencia- } \\
\text { disposición final }\end{array}$} & Diesel & $5,053.08$ & $\mathrm{Kg}$. & COT & $1,581,650$ & $\mathrm{Mg}$ \\
\hline & & $\begin{array}{l}\text { Gasolina } \\
\text { magna }\end{array}$ & 627.30 & $\mathrm{Kg}$. & $\begin{array}{c}\text { Sólidos } \\
\text { suspendidos }\end{array}$ & $2,425,000$ & $\mathrm{Mg}$ \\
\hline & & \multirow{2}{*}{$\begin{array}{l}\text { (Toneladas } \\
\text { residuos)(km } \\
\text { recorridos) }\end{array}$} & \multirow[b]{2}{*}{$24,231,622.80$} & Ton/Km & $\mathrm{CO}_{2 \text {-eq }}$ & $970.11^{*}$ & Ton. \\
\hline & & & & & & & \\
\hline & $\begin{array}{l}\text { Servicio de } \\
\text { recolección }\end{array}$ & $\begin{array}{l}\text { Energía } \\
\text { alterna }\end{array}$ & 323.69 & Kwh & & & \\
\hline \multirow{6}{*}{$\begin{array}{l}\text { Separación } \\
\text { selectiva }\end{array}$} & & $\begin{array}{l}\text { PET } \\
\text { HDPE }\end{array}$ & $\begin{array}{l}1.08213 \\
0.67932\end{array}$ & $\begin{array}{l}\text { Ton. } \\
\text { Ton. }\end{array}$ & & & \\
\hline & & LDPE & 0.358022 & Ton. & & & \\
\hline & & Cartón & 17.64 & Ton. & & & \\
\hline & & Vidrio & 2.63 & Ton. & & & \\
\hline & & Aluminio & 0.4026 & Ton. & & & \\
\hline & & Lamina chilera & 0.7168 & Ton. & & & \\
\hline
\end{tabular}

Fuente: Elaboración propia con base a datos obtenidos de la empresa, así como de fuentes secundarias.

\section{Etapa 3 y 4. Evaluación y análisis del impacto del ciclo de vida}

Al realizar el primer acercamiento del ACV de la actividad realizada del SERC, se obtuvieron los siguientes resultados mostrados en la figura 2, en la cual se hace un análisis comparativo de las categorías de impacto tomando de referencia dos escenarios (Escenario 1 color verde obscuro, Escenario 2 color verde claro). El primero de ellos se realizó solo tomando de referencia la actividad principal de la empresa, mientras que el segundo escenario, se toma en consideración el reciclaje de plástico, cartón-papel, vidrio y aluminio.

Se puede apreciar que la evaluación de los escenarios planteados, el escenario 2, presenta resultados favorables en la categoría de impacto recursos minerales (kg Cu eq 0.06661551 escenario 1 vs -0.03463779 escenario 2). Otras categorías con resultados positivos, pero en menor cantidad, se refleja en el uso de tierra, cáncer humano, eutrofización marítima y de agua TAPIA-IBARRA A. G., VALENCIA-OVALLE A. DEL C., SALDAÑA-DURÁN C. E. 
IMPACTOS AMBIENTALES EN EL SECTOR EMPRESARIAL DE LA RECOLECCIÓN CONTRATADA DE RESIDUOS SÓLIDOS URBANOS EN LA CIUDAD DE TEPIC, NAYARIT, MÉXICO.

dulce. Mientras que en las categorías de impacto calentamiento global, estratósfera, radiación ionizante, formación de ozono, material particulado fino, acidificación terrestre, eco toxicidad terrestre, recursos fósiles y consumo de agua no se presentan resultados favorables.

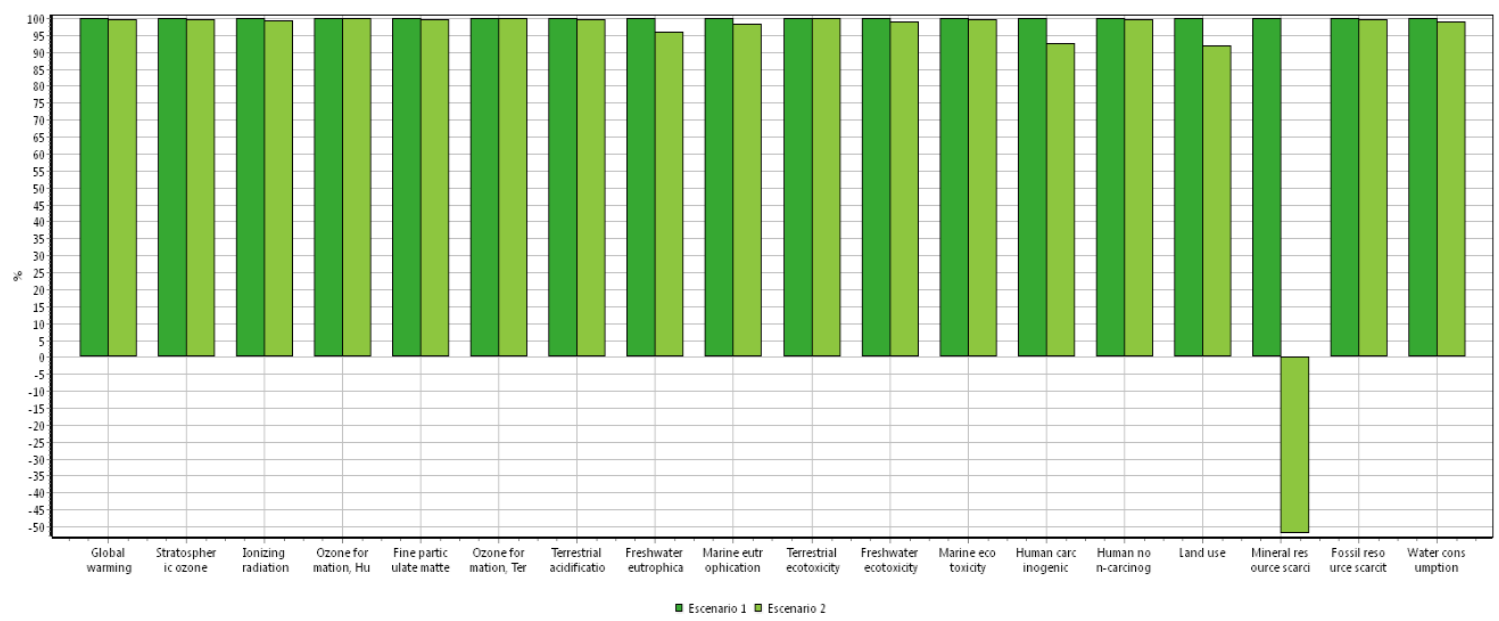

Figura 2. Análisis de impacto tomando dos escenarios posibles.

Fuente: Elaboración propia con ayuda del software SimaPro 8.5.0.

El software SimaPro 8.5.0, permitió realizar la evaluación de cada una de las categorías de impacto, lo que proporcionará un análisis de mayor profundidad. Al realizar el análisis de la categoría calentamiento global, que es una de las categorías con una alta relación con los residuos sólidos urbanos, se determinó que los elementos que impactan en mayor grado es el transporte, tanto para el escenario 1 y 2 , (ver figura 3 ).

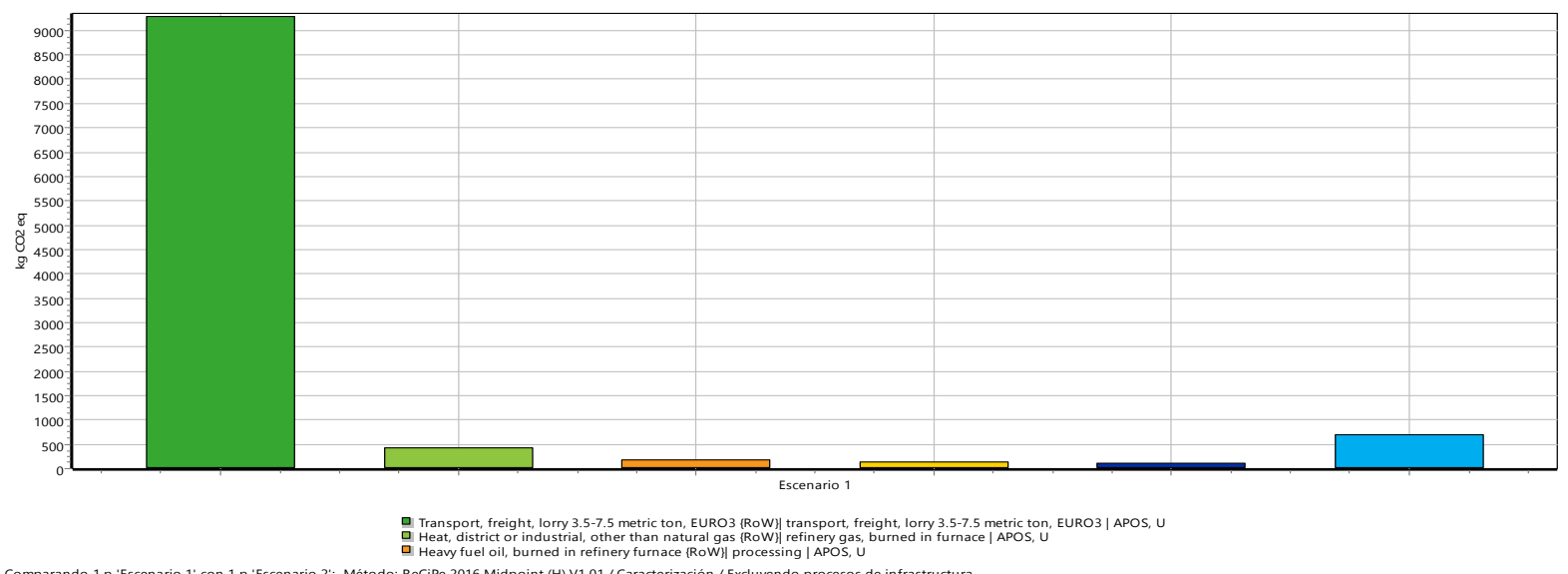

Figura 3. Evaluación categoría de impacto "calentamiento global" escenario 1 y 2.

Fuente: elaboración propia con ayuda del software SimaPro 8.5.0. 
IMPACTOS AMBIENTALES EN EL SECTOR EMPRESARIAL DE LA RECOLECCIÓN CONTRATADA DE RESIDUOS SÓLIDOS URBANOS EN LA CIUDAD DE TEPIC, NAYARIT, MÉXICO.

Conforme a lo establecido en la agenda 21, con relación al cumplimiento de los objetivos para el 2030, la Comisión Económica para América Latina y el Caribe (CEPAL) establece adoptar el reciclaje como alternativa sobre el control de los residuos. Para lograr la gestión sostenible y el uso eficiente de los recursos naturales, así como reducir la generación de residuos a través de prevención, reducción, reciclado y reutilización. Como se muestra en el resultado obtenido (dato

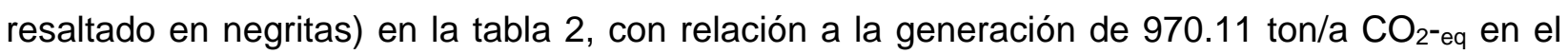
Análisis de Ciclo de Vida, la separación selectiva permite disminuir la generación $\mathrm{CO}_{2}$-eq proveniente de la recolección contratada de 9,744 ton/a de RSM en la Ciudad de Tepic, Nayarit.

De igual forma, Salmerón et al., (2017), determina que las emisiones generadas de 317,173 ton/a de $\mathrm{RSM}$ representan 378,930 ton $/ \mathrm{a} \mathrm{CO}_{2}$-eq $(378.93 \mathrm{Gg})$ destacando que los residuos representan el $5.9 \%$ de las emisiones de $\mathrm{GEI}$ en términos de $\mathrm{CO}_{2}$-eq.

\section{CONCLUSIONES}

Al realizar el primer acercamiento del ACV de la actividad realizada por la empresa (recolección contratada de RSU), se obtuvo un análisis comparativo de las categorías de impacto tomando de referencia dos escenarios. El primero de ellos se realizó sólo tomando de referencia la actividad principal de la organización, mientras que, en el segundo escenario, se toma en consideración el reciclaje de plástico, cartón-papel, vidrio y aluminio; pudiéndose apreciar que la evaluación de los escenarios planteados, el escenario 2, presenta resultados favorables en la categoría de impacto

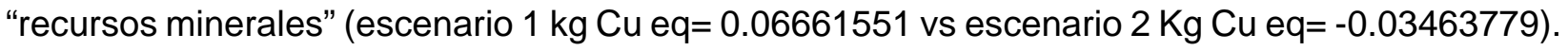
Concluyendo, que el reciclaje debe ser una medida de regulación ambiental en el sector empresarial, especialmente en las organizaciones de recolección contratada, impactando de forma positiva al medio ambiente al reducir significativamente las toneladas de $\mathrm{CO}_{2 \text { eq. }}$ de los gases de efecto invernadero.

Otras categorías con resultados positivos pero en menor cantidad, se refleja en el uso de tierra, cáncer humano, eutrofización marítima y de agua dulce; mientras que en las categorías de impacto calentamiento global, estratósfera, radiación ionizante, formación de ozono, material particulado fino, acidificación terrestre, eco toxicidad terrestre, recursos fósiles y consumo de agua no se presentan resultados favorables, siendo la categoría de impacto "calentamiento global" la que tiene una mayor representatividad. 
IMPACTOS AMBIENTALES EN EL SECTOR EMPRESARIAL DE LA RECOLECCIÓN CONTRATADA DE RESIDUOS SÓLIDOS URBANOS EN LA CIUDAD DE TEPIC, NAYARIT, MÉXICO.

Se determina con la técnica de evaluación del ACV, que los resultados obtenidos indican un futuro brillante para el reciclaje. Los resultados proporcionan una referencia teórica y práctica en la toma de decisión para que el gobierno formule políticas relevantes en atención a la protección al medio ambiente. En resumen, el proceso de reciclaje de residuos de una empresa se tomó como ejemplo de estudio para establecer medidas de regulación ambiental en el sector empresarial, especialmente en las organizaciones de recolección contratada. Además, traería importantes beneficios económicos al incrementar sus ingresos, reduciría los costos derivados del ingreso de los residuos de rechazo al relleno sanitario, impactando de forma positiva al medio ambiente al reducir significativamente las toneladas de $\mathrm{CO}_{2 \text { eq. }}$ de los gases de efecto invernadero.

\section{REFERENCIAS BIBLIOGRÁFICAS}

ANIPAC. (28 de Julio de 2012). Reciclaje de desechos, fuente de ingreso económico y cuidado ambiental. NTR Periodismo Crítico. Recuperado de http://ntrzacatecas.com/2012/07/28/reciclaje-de-desechos-fuente-de-ingreso-economico-ycuidado-ambiental/

AVINA. (2012). Informe Anual 2012. Recuperado de https://www.avina.net/informes-anualesavina/2012.pdf

Hoornweg, D. \& Bhada, P. (2012). What a waste: a global review of solid waste management. Washington, DC: World Bank

Tello, P., Martínez, E., Daza, D., Soulier, M. y Terraza, H. (2010). Informe de la evaluación regional del manejo de Residuos Sólidos Urbanos en América Latina y el Caribe 2010. Recuperado de https://publications.iadb.org/publications/spanish/document/Informe-de-laevaluaci\%C3\%B3n-regional-del-manejo-de-residuos-s\%C3\%B3lidos-urbanos-enAm\%C3\%A9rica-Latina-y-el-Caribe-2010.pdf

Buenrostro, O., Bocco, G. \& Bernache, G. (2001). Urban solid waste generation and disposal in Mexico: a case study. Waste Management and Research, 19(2), 169-76. Recuperado de https://www.ncbi.nlm.nih.gov/pubmed/11721999

Castillo, H. (1983). The garbage society: caciquismo in Mexico City. México: UNAM. 
IMPACTOS AMBIENTALES EN EL SECTOR EMPRESARIAL DE LA RECOLECCIÓN CONTRATADA DE RESIDUOS SÓLIDOS URBANOS EN LA CIUDAD DE TEPIC, NAYARIT, MÉXICO.

Castillo, H. (2003). Garbage work and society. Resources, Conservation and Recycling, 39(3), 193-210. DOI 10.1016/S0921-3449(03)00027-2

Echegaray, F. \& Hansstein, F. V. (2017). Assessing the intention-behavior gap in electronic waste recycling: the case of Brazil. Journal of Cleaner Production, 142(1), 180-190. DOI 10.1016/j.jclepro.2016.05.064

El Financiero. (14 de septiembre de 2016). La basura en México, negocio desaprovechado de 3 mil mdd. El Financiero. Recuperado de http://www.elfinanciero.com.mx/empresas/labasura-en-mexico-negocio-desaprovechado-de-mil-mdd.html

Garcí, M. y Salgado, F. (2007). Reciclado de PET: alternativas de comercialización. Recuperado de http://www.arpet.org/docs/Reciclado-de-PET-Alternativas-de-comercializacion-UBA.pdf

IMNC. (2008). Gestión ambiental-Análisis del ciclo de vida-Principios y marco de referencia. México: Instituto Mexicano de Normalización y Certificación A.C.

INAFED. (2018). Enciclopedia de los Municipios y Delegaciones de México: Nayarit. Recuperado de http://www.inafed.gob.mx/work/enciclopedia/EMM18nayarit/municipios/18017a.html

INECC y SEMARNAT. (2012). Diagnóstico básico para la gestión integral de los residuos. Recuperado de http://biblioteca.semarnat.gob.mx/Documentos/Ciga/libros2009/CD001408.pdf

INEGI. (2015). Cuéntame... información por entidad: Nayarit. Recuperado de http://cuentame.inegi.org.mx/monografias/informacion/nay/poblacion/default.aspx?tema=m $\mathrm{e} \& \mathrm{e}=18$

Jiménez, N. (2015). La gestión integral de residuos sólidos urbanos en México: entre la intención y la realidad. Letras Verdes, 17, 29-56.

Maldonado, L. (2006). Reducción y reciclaje de residuos sólidos urbanos en centros de educación superior: estudio de caso. Revista Ingeniería, 10(1), 59-68. Recuperado de http://www.revista.ingenieria.uady.mx/volumen10/reduccion.pdf

Mattos, D., Gomes, R., \& Ribeiro, G. (2017). Current and future environmental impact of household solid waste management scenarios for a region of Brazil: carbon dioxide and energy analysis. Journal of Cleaner Production, 155(1), 218-228. DOI 10.1016/j.jclepro.2016.05.158 
IMPACTOS AMBIENTALES EN EL SECTOR EMPRESARIAL DE LA RECOLECCIÓN CONTRATADA DE RESIDUOS SÓLIDOS URBANOS EN LA CIUDAD DE TEPIC, NAYARIT, MÉXICO.

Medina, M. (1999). Reciclaje de desechos sólidos en América Latina. Frontera Norte, 11(21), 1231.

Recuperado

de

https://pdfs.semanticscholar.org/0285/74b8532560b36e80f0683db8e00255f268c7.pdf

Medina, M. (2005). Serving the unserved: informal refuse collection in Mexico. Waste Management \& Research, 390-397, 12-31. DOI 10.1177/0734242X05057698

Michell, N. (2007). El reciclaje en el mundo de hoy. Recuperado de http://www.elobservadoreconomico.com/articulo/461

Milenio Digital. (11 de septiembre de 2017). SEMARNAT propone estrategia para tratamiento de residuos sólidos. Milenio. Recuperado de http://www.milenio.com/estados/semarnattratamiento_residuos_solidos-residuos_solidos-electricidad-basura_0_1028297538.html

Moh, Y, \& Manaf, L. (2017). Solid waste management transformation and future challenges of source separation and recycling practice in Malaysia. Resources, Conservation and Recycling, 1-14. DOI 10.1016/j.resconrec.2016.09.012

Olivera, A., Cristobal, S. y Saizar, C. (2016). Análisis de ciclo de vida ambiental, económico y social: una herramienta para la evaluación de impactos y soporte para la toma de decisiones. INNOTEC Gestión, 7, 20-27. Recuperado de https://ojs.latu.org.uy/index.php/INNOTEC-Gestion/article/view/364/pdf_1

Ortega, M. (2011). El reciclaje del PET está en su mejor momento. Tecnología del plástico, 26(4), 12-15. Recuperado de http://www.plastico.com/temas/El-reciclaje-de-PET-esta-en-sumejor-momento+3084014

Parada, J. y Gutiérrez, O. (2015). Desarrollo de nuevas líneas de negocio en la asociación recicladores de tunja "RECITUNJA". En REMINEO, El análisis organizacional en México y América Latina: retos y perspectivas a 20 años de estudios. (pp. 3432). México: Red Mexicana de Investigadores en Estudios Organizacionales.

PNUMA. (2012). Perspectivas del Medio Ambiente Mundial: opciones de política para américa Latina y el Caribe. Panamá: Novo Art.

Torres, A., Salas, L. y García, L. (2010). Oportunidades de mercado para empresas recicladoras de plástico en China. Red Internacional de Investigadores en Competitividad, 4(1), 491-510. Recuperado de https://www.riico.net/index.php/riico/article/view/713/649

TAPIA-IBARRA A. G., VALENCIA-OVALLE A. DEL C., SALDAÑA-DURÁN C. E. 
IMPACTOS AMBIENTALES EN EL SECTOR EMPRESARIAL DE LA RECOLECCIÓN CONTRATADA DE RESIDUOS SÓLIDOS URBANOS EN LA CIUDAD DE TEPIC, NAYARIT, MÉXICO.

Salas J. y Quesada, H. (2006). Impacto ambiental del manejo de desechos sólidos ordinarios en una comunidad rural. Revista Tecnología en Marcha, 19(3), 9-16. Recuperado de https://revistas.tec.ac.cr/index.php/tec_marcha/article/view/3/2

Salmerón, Y., Cabrera, R., Juárez, A., Sampedro, M., Rosas, A. J. y Rolón, J. (2017). Emisiones de gases de efecto invernadero en vertederos de residuos sólidos urbanos. Iberoamericana de Ciencias, 69-79. Recuperado de http://www.reibci.org/publicados/2017/feb/2100108.pdf

SEMARNAT. (2008). Programa nacional para la prevención y gestión integral de los residuos. México: SEMARNAT.

SEMARNAT. (2010). Directorio de centros de acopio de Materiales provenientes de residuos en México 2010.

Recuperado

de http://www.semarnat.gob.mx/archivosanteriores/transparencia/transparenciafocalizada/res iduos/Documents/directorio_residuos.pdf

SEMARNAT. (2012). Compendio de estadísticas ambientales 2012: tema residuos sólidos urbanos. Recuperado de http://app1.semarnat.gob.mx/dgeia/Compendio_2012/mce_index.html

SEMARNAT. (2012). Informe de la situación del medio ambiente en México. Recuperado de https://apps1.semarnat.gob.mx:8443/dgeia/informe15/tema/pdf/Informe15_completo.pdf

Tisserant, A., Pauliuk, S., Merciai, S., Schmidt, J., Fry, J., Wood, R., \& Tukker, A. (2017). Solid waste and the circular economy: a global analysis of waste treatment and waste footprints. Research and Analysis, 628-640. DOI 10.1111/jiec.12562

Vélez, M. (2014). Elaboración del inventario para el análisis de ciclo de vida de suelos adicionados con polímeros y su aplicación para la estabilización de vías terciarias. Medellín: Universidad de Medellín. 